\title{
A systematic review and meta-analysis protocol of observational studies to assess the association between sleep and mental resilience in healthy individuals
}

\author{
Asma Alamoodi \\ Zayed University \\ lan Grey \\ Lebanese American University \\ Linda Östlundh \\ United Arab Emirates University \\ Omar M. Omar \\ University of Birmingham \\ Michael Grandner \\ University of Arizona
}

Teresa Arora ( $\nabla$ teresaarora8@gmail.com )

Zayed University https://orcid.org/0000-0001-8360-7358

\section{Protocol}

Keywords: sleep duration, sleep quality, mental resilience, psychological resilience, systematic review, meta-analysis, protocol.

Posted Date: October 15th, 2020

DOI: https://doi.org/10.21203/rs.3.rs-48076/v2

License: (c) (i) This work is licensed under a Creative Commons Attribution 4.0 International License. Read Full License 


\section{Abstract}

Background: Mental resilience has emerged as a recent focus within the context of positive psychology. Resilience is a potentially modifiable construct. Some preliminary evidence from experimental studies as well as clinical populations has indicated that sleep may be related to mental resilience, but little is known about this relationship in healthy populations. Sleep is a complex with multi-factorial outcome, although the majority of research effort has focused on sleep duration and sleep quality. Our systematic review and meta-analysis will identify original research data from observational studies to assess if sleep duration and/or sleep quality is associated with levels of psychological resilience amongst healthy children, adolescents and adults. We hypothesize that there will be a positive association between sleep duration/quality and mental resilience across all age groups.

Methods: A comprehensive, systematic search for literature will be conducted in June 2020 (LÖ), covering a total of eight academic databases (PubMed, Embase, Psyclnfo, PsycArticles, CINAHL, Scopus, Web of Science and Academic Search Complete). Sources for grey materials will also be included and experts in the field will also be contacted for inclusion of unpublished data. PubMed and PubMed's MeSH terms were used to develop the search strategy and systematically identify relevant search terms. Two authors (IG \& AA) will screen the articles independently, and a third researcher (TA) will resolve discrepancies. The primary outcome is mental/psychological resilience. The systematic review will adhere to the Preferred Reporting Items for Systematic Reviews and Meta-Analyses (PRISMA) guidelines.

Discussion: The results of our systematic review and meta-analysis are intended to be published in an appropriate scientific peer-reviewed journal and will be made publicly available. The findings from our project are envisioned to inform and educate the public as well as healthcare and education systems, globally. Our systematic review protocol has been peer-reviewed and is currently registered in the international database, PROSPERO.

Systematic review registration: CRD42020191119 (submitted on 2 July 2020).

\section{Background}

Mental resilience relates to an individual's ability to successfully mentally and/or emotionally adapt to challenging and stressful situations and to return to normal functioning in a timely manner after facing such situations. It is viewed as an active process which results in positive adaptation and recovery in response to adversity.(1) Thus, individuals who lack the necessary psychological and/or behavioural capabilities to positively adapt to stressful circumstances are considered to have low levels of mental resilience which may be associated with an increased vulnerability for the development of a range of mental health difficulties. Stress is commonplace in contemporary society and is a customary reaction to adversity. However, dysfunctional stress reactivity can result in the manifestation of several psychological disorders including major depressive disorder (MDD), post-traumatic stress disorder (PTSD), and/or other anxiety disorders.(2) Some individuals may possess intrinsic mechanisms which 
can enhance resilience to stress, thus demonstrating adapting to stress in the absence of developing psychopathology.(2) Higher levels of resilience may also delay the aging process as well as improve quality of life and overall health and wellbeing.(2)

A recent systematic review of nurses working in mental health settings (where stress is commonplace) found that low-moderate levels of resilience were correlated with depression and burnout.(3) Conversely, higher levels were linked to more positive outcomes including hardiness, self-esteem as well as enhanced job and life satisfaction.(3) Similar observations have also been documented amongst corporate executives where higher resilience was positively associated with quality of life and lower resilience was negatively associated with perceived stress and subjectively reported mental health conditions.(4) Similar patterns have been observed amongst clinical populations. For example, one study of 58 insomnia patients and 38 healthy controls revealed that patients with insomnia had lower levels of resilience, higher levels of stress, increased emotion dysregulation, higher stress-related sleep reactivity and presleep cognitive hyperarousal compared to the healthy controls, even after adjustment for common comorbid psychological symptoms.(5) Recently, resilience has also been associated with perceptions of health as well as immune function.(6) A large sample $(n=779)$ of participants were asked to complete a battery of questionnaires pertaining to resilience, perceived health and immune function. After adjustment for a wide range of confounders, the authors observed a significant positive correlation between resilience and perceived immune status $(r=0.124, p<0.01)$ as well as perceived health $(r=0.233$, $p<0.001)$. Whilst the relationships between mental and physical health appear to be well-established in relation to mental resilience, a deeper understanding is needed surrounding the factors that contribute to this psychological construct.

Mental resilience has emerged as a focus of interest within the context of positive psychology. It may represent a modifiable characteristic, in that resilience can be developed by intentionally adapting emotional thoughts and actions in response to specific triggers.(7) This is reflected in the development of specific intervention approaches such as strength-based cognitive behavioural therapy (SB-CBT) programmes to target resilience building.(8) More recently, attention has turned to the role of specific factors in the promotion of resilience such as sleep. In one key study, 213 young healthcare professionals (mean age 28 years) completed a cross-sectional survey responding to questions about sleep, resilience, perceived stress, health status, mindfulness and self-compassion.(9) In addition to other observations, increased disturbances to sleep were found to be significantly correlated with lower levels of resilience $(r=-0.24, p<0.001)$. While a substantial body of research indicates the central role of sleep in relation to various aspects of mental and physical health, this novel finding would suggest that sleep may have important implications in terms of resilience and understanding the intricate relationship between these two factors which are known promoters of wellbeing. In addition, it suggests that sleep health supports positive and adaptive mental health, in addition to protecting against adverse outcomes. To date, at least one attempt has been made to causally examine the relationship between sleep and resilience. In a 24-hour total sleep deprivation study, greater expressed neuroendocrine hormone activity of rennin, angiotensin II and cortisol were observed in participants with low resilience.(10) Thus, it is likely that sleep may be a vital component that is closely connected to mental resilience. In order to disentangle 
and better understand the role of sleep in relation to mental resilience, it is first important to identify the putative relationship between these two aspects amongst healthy individuals at a population level. If our findings establish an association between sleep and resilience, then we recommend further additional reviews to assess the same relationship amongst specific clinical populations. Future studies can then consider developing and delivering interventions to enhance levels of resilience and sleep improvement. In turn, this may result in downstream effects on optimal wellbeing, minimise the onset and progression of chronic health conditions, foster stronger immune function, and potentially prolong longevity.

\section{Methods/design}

Our systematic review and meta-analysis has been developed to assess the potential relationship between both sleep duration and sleep quality in relation to mental/psychological resilience across healthy paediatric and adult populations, drawn from the general population. Our findings are intended to inform healthcare providers, public health, education systems and the general population about the importance of sleep in relation to mental/psychological resilience, should we identify a relationship between these factors.

This protocol was designed in accordance with the preferred reporting standards for systematic review protocols (PRISMA-P), which have been previously established in the PRISMA-P administrative information checklist.(11) Our study protocol was submitted to the international prospective register of systematic reviews (PROSPERO) on 2 July 2020. Our protocol has been peer-reviewed and is registered on PROSPERO (CRD42020191119).

\section{Eligibility criteria}

All components of participants, intervention, comparator, and outcome (PICO), consistent with PRISMA-P reporting guidelines(11), are as follows:

\section{Participants}

Our systematic review targets the inclusion of healthy humans drawn from the general population. Articles involving any of the following populations will be excluded:

1. Animals;

2. Infants (up to 12 months);

3. Clinical populations including those with existing diagnosed sleep disorders, mental health disorders, and/or physical disabilities.

Intervention 
Our systematic review is focused on the relationship between sleep duration/quality and mental/psychological resilience. No intervention/exposure is applicable for our review.

\section{Comparator}

Our systematic review will capture articles that assess the relationship between sleep quality and sleep duration in relation to psychological resilience. Primary comparison will be those with good versus poor quality sleep, in relation to the outcome. Secondary comparison will be to compare individuals with recommended sleep duration (based on age by expert consensus) to either short or long sleepers, depending on the articles retrieved. In the meta-analysis, the statistical information will be pooled and presented according to the above, where possible.

\section{Outcome}

The primary outcome of our systematic review and meta-analysis is mental/psychological resilience.

\section{Type of studies}

We will screen and identify observational studies only and include cross-sectional, prospective and survey-based study designs. For studies that obtain information pertaining to sleep duration/quality and mental/psychological resilience but do not report on the direct relationship between the two variables, we will contact the corresponding author up to three times by email to request the data before excluding the full-text article. Published conference abstracts presenting original research data will also be included, based on recent recommendations.(12) Qualitative studies, case studies, protocol studies, questionnaire validation studies, experimental studies and in vitro/genetic studies will be excluded from the review. Furthermore, reviews of any kind, commentaries, editorials, reports, correspondence will not be eligible, unless they present original research data on the area of interest.

\section{Information sources}

A comprehensive, systematic search for literature will be conducted in June 2020, covering a total of eight academic databases (PubMed, Embase, Psyclnfo, PsycArticles, CINAHL, Scopus, Web of Science and Academic Search Complete). Sources for grey materials will also be included. Pre-searches to identify relevant information sources and search terms were performed in March-June 2020. PubMed and PubMed's MeSH terms were used to develop the search strategy and systematically identify relevant search terms.

\section{Search strategy}

A pre-screening of the preliminary result from PubMed was conducted as a support for finalizing the search strategy:

((resilience*[Title/Abstract] OR "mental toughness" [Title/Abstract] OR hardiness[Title/Abstract] OR flourishing [Title/Abstract] OR "Resilience, Psychological"[Mesh]) AND (sleep*[Title/Abstract] OR awake* 
The PubMed search will be adapted and applied to included databases. The final search strategy will be developed by a medical librarian (LÖ) in close collaboration with subject specialists (TA, IG \& MG). All search terms will be searched in a combination of the fields: "title", "abstract" and in the "MeSH"/"thesaurus" when available. No filters or limitations will be added to ensure the inclusion of preindexed materials. All databases and grey sources will be searched from their inception to June 2020 . Hand screening of reference lists for each included paper will be conducted. Experts in the field will also be contacted for any unpublished data which may be eligible for inclusion.

\section{Study records}

\section{Data management}

The records identified in the literature search will be uploaded to the systematic review software Covidence (Veritas Health Innovation, 2020) for automatic de-duplication and blinded screening. A search log with detailed search strings, source coverage, search dates, result and notes for all information sources will be appended to the review.

\section{Selection process}

Covidence software will be used to detect and exclude any duplications, which will be managed by LÖ. The remaining articles that potentially meet our eligibility criteria will first be screened by title and abstract by two independent reviewers (IG \& AA). Articles will either be accepted or rejected by each of the two reviewers and any discrepancies will be resolved by a third reviewer (TA), based on the pre-defined inclusion/exclusion criteria described. Following title and abstract screening, the full text of the remaining articles will be sourced by LÖ and uploaded into Covidence software for review by the same two independent reviewers (IG \& AA). Any discrepancies will be resolved by a third reviewer who has not been involved in the full text screening process (TA). Cabell's Blacklist for predatory journals will be informed to confirm the scientific status of all included papers published in open access journals.

\section{Data collection process}

For articles that fulfil the pre-defined inclusion criteria, two authors (IG \& TA) will independently extract relevant data using a data extraction sheet. Data extraction will be performed using Excel. In case of any discrepancies, a third reviewer (OMO) will resolve them by discussion until a consensus is reached. The final data extraction will also be overseen and checked by a statistician (OMO). The corresponding author of any article, where additional data needs to be obtained, will be contacted by email up to three times and responses will then be recorded and archived.

\section{Data items}

The data extraction sheet will encompass a range of information including the following: 
1. First author's surname;

2. Year of publication;

3. Sample size;

4. Country where study was conducted;

5. Gender split;

6. Age of the sample;

7. Tool(s) used to assess resilience/hardiness/mental toughness (study outcome);

8. Tool(s) used to assess sleep duration/quality;

9. Confounders that were included for adjustment;

10. Effect size reported for the relationship between sleep and resilience.

\section{Risk of bias in individual studies}

Risk of bias assessment will be performed by two reviewers (IG \& TA). MG will later resolve any discrepancies through discussion until a consensus is reached. The Study Quality Assessment Tool for Observational Cohort and Cross-Sectional Studies from the National Institutes of Health,(13) or the Newcastle-Ottawa Scale for non-randomized studies(14) will be used to assess the quality and bias of included studies.

\section{Data synthesis}

The results of the included studies will be summarised in a table which depicts all of the data extraction information. Additionally, a detailed narrative synthesis of the findings will be written where we discuss methodology in detail along with discussing possible explanations for differences in effect sizes that are observed. A meta-analysis will be later conducted where the effect sizes from suitable studies are combined using a random-effects model. This will be conducted by the team's statistician (OMO) to assess the strength of the body of evidence.

\section{Discussion}

Systematic reviews do not require ethical approval due to the type of study design. The results of our systematic review are intended to be published in an appropriate scientific peer-reviewed journal and will be made publicly available. The study findings will also be disseminated through printed copies from the accepting journal as well as through online platforms. The findings are intended to be disseminated, not only to the scientific community, but also to the general public, public health officials, as well as healthcare and education systems. Our vision is to inform and raise awareness about the potential relationship between sleep and resilience to a wide audience.

\section{Abbreviations}


PRISMA=Preferred Reporting Items for Systematic Reviews and Meta-Analyses

MDD=Major Depressive Disorder

PTSD $=$ Post-Traumatic Stress Disorder

SB-CBT =Strength-Based Cognitive Behaviour Therapy

PICO=Participants, Interventions, Comparators, and Outcomes.

\section{Declarations}

Ethics for approval and consent to participate: Not applicable. Systematic reviews do not require ethical approval due to the type of study design.

Consent for publication: Not applicable.

Availability of data and materials: The dataset which will be developed for our systematic review and meta-analysis will be drawn from combining data from published studies which are publicly available. The eventual dataset used will be made available from the Corresponding Author on reasonable request.

Competing interests: The authors declare that there have no competing interests.

Funding: There is no specific grant for this research from any funding agency. Zayed University is the sponsor of this review but has not provided any direct funding support.

Authors' contributions: TA conceived and designed the study and is the primary author. TA and AA drafted the protocol manuscript. IG \& AA will screen the articles retrieved from the search and TA will resolve all screening discrepancies. LÖ derived and conducted the full search strategy for each database and inclusion of various terms were supported by TA, IG, and MG. OMO is the team statistician and will oversee the data extraction process as well as conduct the meta-analysis. MG is the corresponding author and will provide feedback on the study protocol design and critically revise earlier versions of the manuscript for intellectual content. MG serves as guarantor for the review.

Acknowledgements: Given that we will include articles written in any language, we would like to acknowledge individuals who kindly help with translation of any foreign language articles which are outside of the research team's language capability.

\section{References}

1. Rolin H, Fossion P, Kotsou I, Leys C. [Perspectives on resilience : trait or aptitude ?]. Rev Med Brux. 2018;39(1):22-8.

2. Faye C, McGowan JC, Denny CA, David DJ. Neurobiological Mechanisms of Stress Resilience and Implications for the Aged Population. Curr Neuropharmacol. 2018;16(3):234-70. 
3. Foster K, Roche M, Delgado C, Cuzzillo C, Giandinoto JA, Furness T. Resilience and mental health nursing: An integrative review of international literature. Int J Ment Health Nurs. 2019;28(1):71-85.

4. Kermott $C A$, Johnson RE, Sood R, Jenkins $S M$, Sood $A$. Is higher resilience predictive of lower stress and better mental health among corporate executives? PLoS One. 2019;14(6):e0218092.

5. Palagini L, Moretto U, Novi M, Masci I, Caruso D, Drake CL, et al. Lack of Resilience Is Related to Stress-Related Sleep Reactivity, Hyperarousal, and Emotion Dysregulation in Insomnia Disorder. J Clin Sleep Med. 2018;14(5):759-66.

6. Van Schrojenstein Lantman M, Mackus M, Otten LS, de Kruijff D, van de Loo AJ, Kraneveld AD, et al. Mental resilience, perceived immune functioning, and health. J Multidiscip Healthc. 2017;10:107-12.

7. Iacono MV. Promote a Healthy Workplace: Build Resilience. J Perianesth Nurs. 2017;32(1):81-2.

8. Padesky CA, Mooney KA. Strengths-based cognitive-behavioural therapy: a four-step model to build resilience. Clin Psychol Psychother. 2012;19(4):283-90.

9. Kemper KJ, Mo X, Khayat R. Are Mindfulness and Self-Compassion Associated with Sleep and Resilience in Health Professionals? J Altern Complement Med. 2015;21(8):496-503.

10. Sun X, Dai X, Yang T, Song H, Yang J, Bai J, et al. Effects of mental resilience on neuroendocrine hormones level changes induced by sleep deprivation in servicemen. Endocrine. 2014;47(3):884-8.

11. Moher D, Stewart L, Shekelle P. Implementing PRISMA-P: recommendations for prospective authors. Syst Rev. 2016;5:15.

12. Scherer RW, Saldanha IJ. How should systematic reviewers handle conference abstracts? A view from the trenches. Syst Rev. 2019;8(1):264.

13. National Heart Lung and Blood Institute. Study Quality Assessment Tools [Available from: https://www.nhlbi.nih.gov/health-topics/study-quality-assessment-tools.

14. Wells GA, Shea B, O'Connell D, Peterson J, Welch V, Losos M, Tugwell P. The Newcastle-Ottawa Scale (NOS) for assessing the quality of nonrandomised studies in meta-analyses [Available from: http://www.ohri.ca/programs/clinical_epidemiology/oxford.asp.

\section{Supplementary Files}

This is a list of supplementary files associated with this preprint. Click to download.

- PRISMAPchecklist.docx 